\title{
A PRACTICAL METHOD FOR THE MEASUREMENTS OF THE CHAOTICITY OF ELECTRIC AND MAGNETIC BRAIN ACTIVITY
}

\author{
ZBIGNIEW J. KOWALIK and THOMAS ELBERT \\ Institute of Experimental Audiology, Center for Biomagnetism, \\ University of Münster, D-48129 Münster, FRG \\ Department of Psychology, University of Konstanz, D-78434 Konstanz, FRG
}

\begin{abstract}
The degree of complexity of the cortical processes related to different cognitive actions should logically produce an image in the electric and magnetic signals of the human brain. Hence, those measures of chaoticity will depend on the particular task being investigated. In many cases, the duration of a brain process does not allow for the generated signal to be treated as stationary. Therefore, the application of the standard method of nonlinear system theory is often questionable. The chaoticity of the process has to characterize the predictability of future state of the system. In the stationary case, such a quantity can be directly expressed by the largest Lyapunov exponent or by $\mathrm{K}-\mathrm{S}$ entropy.

In this study, we performed a test for the applicability of the local Lyapunov exponent for the description of the chaoticity of the brain processes measured in EEG and MEG experiments.' We demonstrate an algorithm for computation of chaoticity based on the local Lyapunov exponent and present possible applications of this method for specific cases with a diagnosis of schizophrenia or of tinnitus. We also show that chaoticity is able to detect critical transitions (phase-transition-like phenomena) which occur in the dynamics of neural mass activity at a specific point in time.
\end{abstract}

\section{Introduction}

Certain information provided by experimental times series can only be extracted by analyzing the dynamical characteristics. However, the first limitation for using such procedures are the ubiquitous environmental and instrumental noise and, in addition, the intrinsic noise of the system under investigation. Signal resolution and sampling rate A/D conversion also influence the estimation of dynamical quantities like fractal dimension, KolmogorovSinai entropy, Lyapunov exponent, etc. Despite such limitations it has become possible to characterize in some cases the dynamics of EEG (Electroencephalogram) and MEG (Magnetoencephalogram) which are produced by a given physiological and psychological condition. Such a quantification usually focuses on the correlation dimension of the experimentally reconstructed attractor [Grassberger \& Procaccia, 1983a], the deterministicity measure [Kaplan \& Glass, 1992] and in order to estimate the predictability of EEG/MEG time series, the entropy or Lyapunov exponents. With respect to clinical applications the most interesting measure is the predictability which formally results from the solution of the stability problem, i.e., the spectrum of Lyapunov exponents [Farmer et al. 1983]. Although all mentioned quantities are linked in an idealistic system, they provide independent information when extracted from experimental data bases. 
In this paper, we will discuss various ways of implementing measures of chaos theory in studies of nonstationary or not strictly stationary systems, such as spontaneous EEG and MEG or other physiological times series. One important question concerns the predictability and the deterministicity of the system state, as these characteristics are required to justify the analytical implementations derived from the theory of nonlinear dissipative systems. The predictability in low-dimensional systems is inversely proportional to the Largest Lyapunov Exponent (LLE) only in cases where stationarity can be assumed. For higher-dimensional but still stationary systems, the LLE can be replaced by the Kolmogorov-Sinai entropy. Unfortunately, we cannot assume a stable attractor for neural mass activity and, therefore, we are not able to reach final conclusions concerning brain dynamics. Therefore, we need to find a measure which characterizes the system's momentary stability or the variation in its information capacity. For this purpose, we introduce a chaoticity measure which fulfills these conditions and, hence, may prove useful for the analysis of time series in biology and medicine.

\section{Stability and the Spectrum of Lyapunov Exponents}

An ergodic, nonlinear, and deterministic system with at least one positive LE is by definition chaotic [see also Farmer et al., 1983]. Therefore, when studying nonlinear dynamics, one must first verify whether these conditions are fulfilled. Ergodicity can be determined by sequentially examining the distribution of the Fourier components: If it does not change - the system is ergodic. Nonlinearity can be tested by applying surrogate data set test [Theiler et al., 1991] or through redundancies [Paluš et al., 1993]. Finally, the deterministicity of the signal can be uncovered by using the test for determinism [Kaplan \& Glass, 1992]).

One method of determining the stability of a system is to disturb it by altering one of its variables and by following its subsequent response. A given system in one of its stable states should return to its original state after a small pertubation. This process is one of relaxation with a convergence that follows an exponential function. For unstable conditions, the system diverges exponentially from the original state. The evolution of the disturbance $\delta_{0}=\delta(t=0)$ in the consecutive time points is described by: $\delta_{n}=\delta_{0} \exp (\lambda n)$. It is evident that $\delta$ approaches zero with an increasing time $(n)$ for negative $\lambda$ and $\delta$ approaches infinity if $\lambda$ is positive. This formulation of the stability shows us that an $N$-dimensional dynamical system consists of multidirectional $N$-values of the Lyapunov exponent (LE). However, this set of values, known as the spectrum of Lyapunov Exponents, does not consist of all possible combinations of LE values. Because the system is a priori dissipative, the sum of all LE must be negative (the phase space volume must be shrinking), i.e., there exists at least one LE which is negative. More interesting, of course, is the value of the largest LE (LLE): If an LLE is positive the system is expanding in one direction, neighboring trajectories are diverging and, consequently, the system is chaotic. Moreover, the greater the value of the LE the shorter the predictability across time and the larger the chaoticity.

Although it may be possible to perform an experiment with a repetition of measurements such that close trajectories will be realized, it is rather difficult to forsee such an experiment for physiological systems. In order to measure the LE, we must search for nearby trajectories in already measured data and reconstruct the time evolution of separations between them. Repeating this process for many pairs of trajectories, we can estimate an average logarithmic ratio of the developed distances which characterize the stability of the system locally in time and phase space. This number is negative for periodic signals, zero for quasiperiodic or intermittent, and positive for a chaotic motion, respectively. In $N$-dimensional space, LEs are equal to the length of axes of the $N$-hyperellipsoid (in $1 \mathrm{D}$ this is an interval, in 2D this is an ellipse, in 3D ellipsoid, etc.). The LLE, having the longest axis, determines the direction in space where maximal volume changes occur as time progresses, i.e., the direction of maximal instability. To find values of LEs one must perform this procedure for a scalar time-series $\{X\}$ measured. Applying Taken's theorem [Takens, 1981], we can reconstruct an attractor with the same topological features as the original attractor. ${ }^{1}$ The reconstruction employs the time-lag technique yielding the state vectors in the following form:

$$
\{x(t), x(t-\tau), \ldots, x(t-(e-1) * \tau)\}
$$

\footnotetext{
${ }^{1}$ Here it is made evident that this should be called a building or a construction process of the analogous attractor rather than a process of reconstruction.
} 
where the delay-time $\tau=p^{*} d t$, with $d t$ being the sampling interval, $t \in[d t, N * d t], p, e \in I$. The value of $e$ has to be large enough to be embeded in the phase space: $e \geq 2 n+1$, where $n$ is the amount of the state variables, and $N>10^{n}$ [Eckmann \& Ruelle, 1992]. In general, the parameters required for the reconstruction are not known and we are never guaranteed of its correctness. The requirement of the big $N$-value is possible for only well controlled systems, generally meaning not in vivo observations, so that for the reconstruction one has to guess both parameters: delay-time $\tau$ and embedding dimension $e$. For very simple dynamical systems, there is usually one characteristic maximum in the power spectrum at $\omega_{0}$, so that one can use $\tau=\frac{\pi}{2 \omega_{0}}$ as the delay time (see also Destexhe et al. [1988]). The most frequently applied method is the first zero-crossing of autocorrelation function [Schuster, 1989]. Another method searches for the minimum in the mutual information [Fraser \& Swinney, 1986; Fraser, 1989] (see also Graf \& Elbert [1989] for an application to EEG, suggesting that it may be sufficient to consider the autocorrelation). Theoretically, for the infinite time series the choice of the delay-time is unimportant and the key role plays the choice of embedding dimension $e$. In practice, the measured signals are short and the choice of both parameters is critical. The choice of the embedding dimension is usually carried out quite pragmatically by the estimation of the correlation dimension for different values of $e=3,4, \ldots E$. Here, one must assume that if the correlation dimension converges at some $E$-value then this value corresponds to the proper embedding. There are several methods which allow (at least for the known basic examples and disposing complete data sets) for the generation of an attractor which is similar in character to the original one (see, for example, Buzug et al. 1990]).

Based on the reconstructed phase space, it is possible to estimate the average rate of the evolution of a chosen small volume element (multidimensional ellipsoid). The LLE is then defined as:

$$
\lambda_{1}=\lim _{t \rightarrow \infty} \frac{1}{t} \log \left(\frac{\operatorname{dist}(t)}{\operatorname{dist}\left(t_{0}\right)}\right),
$$

where dist $(t)$ denotes the length of the longest (principal) axes of the elipsoid at time $t$ and $\operatorname{dist}\left(t_{0}\right)$ denotes the initial disturbance, repectively. The first practical limitation of this definition is trivial: We cannot fulfill the requirements of an infinite time series: $t=N d t$ and both $N$ and $d t$ are finite. Never- theless, if the system conserves its total energy, and if the analysed time series is long enough to more or less reconstruct the attractor, the estimated value will be close to the theoretical one. Practical methods for the estimation of the LE from experimental data sets have been adequately described in recent literature [Wolf et al., 1985; Sano \& Sawada, 1985; Sato et al., 1987; Eckmann et al., 1986; Brown et al., 1991; Rosenstein et al., 1993].

Our method is based on the definition of Lyapunov exponents as presented by Wolff [1992] (see also the interpretations by Mayer-Kress \& Layne [1987b] and Wolf et al. [1985]). To estimate the LLE one must first estimate partial Lyapunov exponents for a given pair of points in space; namely, for the point $X_{i}$ and $\log m$ :

$$
\lambda_{i, m}=\frac{1}{2 m k_{i} \log 2} \sum_{j \in S_{i}} \log \frac{\operatorname{dist}\left(X_{i+m}, X_{j+m}\right)}{\operatorname{dist}\left(X_{i}, X_{j}\right)}
$$

where

$$
\operatorname{dist}\left(x_{i}, x_{j}\right)=\sum_{k=1}^{e}\left(x_{i+k * \tau}-x_{j+k * \tau}\right)^{2}
$$

is the square of distance between trajectories in the moment $t$, and $S_{i}$ is a set of points with distance smaller than some arbitrary value and $k_{i}$ is the amount of these distances, respectively.

Repeating this procedure for $N$ pairs and averaging this, i.e.,

$$
L(t, N)=\left\langle\lambda_{i}(t)\right\rangle_{N}
$$

should yield the Largest Lyapunov Exponent if $N$ is a large number and only if the system dynamics do not change.

\section{Kolmogorov-Sinai Entropy}

As previously mentioned, chaoticity can be evaluated by both the largest Lyapunov exponent and by the Kolmogorov entropy. Both measures characterize similar properties, though they rely on different algorithms. The entropy is strictly connected to the classical Shannon information capacity. Suppose, for instance, that we have a physical system which can assume $N$ different uncorrelated states. For every one of these states some chosen domain can be realized $n_{i}$ times. Thus, the probability that the $i$ th state occurs is $p_{i}=n_{i} / N$. The information 
capacity is then defined as:

$$
I=-\sum_{i}^{N} p_{i} \log p_{i}
$$

The entropy of a given deterministic process is defined as the probability of the respective trajectory crossing the $i$ th region of the phase space. If we denote this probability as $p_{i}$, then the sum of all products $p_{i} \log p_{i}$ characterizes an average information loss in space (where $i$ is an index numbering all smaller subspaces). The only difference between information gain $I$ and entropy $K 2$ is the base of the logarithmic function. The $K 2$-entropy in a stationary chaotic system is strictly connected to the Lyapunov exponents: the sum of all positive LE equals $K 2$ [Pesin, 1977]:

$$
K 2=\sum_{\left\{j: \lambda_{j}>0\right\}} \lambda_{j} .
$$

The wide spectrum of applications of the entropy and methods of its estimation has previously been thoroughly described in the literature [Grassberger and Procaccia, 1983b; Györgyi and Szèpfalusy, 1985; Mayer-Kress, 1986] For the purposes of this paper we will only exemplify the application of the ratings of the $K 2$-entropy to MEG analysis.

\section{The Question of Nonstationary Behavior}

It is clear that spontaneous EEG and MEG signals measured during an uncontrolled task do not remain stationary long enough to provide sufficient data for reliable estimation. Some investigators [Freeman \& DiPrisco, 1986; Mayer-Kress \& Holzfuss, 1987; Ellner, 1988] have suggested that these mutually exclusive requirements represent a fundamental limitation in the application of chaos theory to biological systems. Theoretically, there are two possible treatments of the nonstationarity problem. One is to use very short time intervals such that the dynamics remain unchanged. The other is to use very long time intervals where the average energy remains constant. Unfortunately, when applied to EEG/MEG, the latter solution unrealistically assumes a constant brain state for the duration of the measurement period. Therefore, the only solution is to use short epochs (i.e., epochs which must still be presumed to be stationary). Consequently, large errors in estimation arise due to the small amount of data. However, these errors will be systematic, and the estimates can still be applied for the experimental comparative studies.

Using data recorded from the olfactory bulb of the rabbit, Skinner and associates [Skinner et al., $1990 ; 1992$ ] found that epochs as short as $500 \mathrm{msec}$ were necessary to achieve statistical stationarity. Following a novel odour, the $500 \mathrm{msec}$ epochs still appeared to be stationary within this condition, but now the fractal dimension estimates were observed to be significantly increased compared to control. Similarly, Rapp and associates [Rapp et al., 1990] proposed that human EEG epochs of $1 \mathrm{~s}$ remain stationary, demonstrating that the D2-dimension values following a target stimulus (active reaction on driver) were significantly lower than those following a nontarget stimulus (passive receiving).

The problem arising with these brief-interval studies is that the post-stimulus dimensions may appear stationary, although in reality they are not, i.e., the dimensional shift may not change to a new stable state, but may actually undergo a variety of rapid nonstationary changes.

One approach to the solution of this problem of data nonstationarity could be to apply a method of continuous estimation of the dimension, with the expectation that the reference vector (i.e., the "point"), which spans only a short interval, would remain stationary and would dominate the calculations, making the overall estimate less sensitive to nonstationarities [Farmer et al., 1983; Soong \& Stuart, 1989; Skinner et al., 1990]. When the pointwise scaling dimension (D2) was applied to biological data (EEG, EMG, heartbeat intervals) it was found to have a considerable error of estimation. This error could have arisen because the method still required data stationarity over the whole epoch. Another method, called the point-D2 (PD2), was developed to circumvent the requirement for data stationarity. In this method, only selected vectordifference lengths are used to determine the dimensional estimate for a particular data point; the selection is based on an algorithm which determines for each reference vector a stationary subepoch [Skinner et al., 1990; 1992]. It has been demonstrated that this deterministic measure (i.e., the PD2) is inherently more sensitive to the output of the underlying biological system than a stochastic measure (e.g., the signal-averaged mean [Freeman \& Skarda, 
1985]) or the standard deviation [Freeman, 1979] applied to the same data.

The above mentioned considerations explain why the values of the chaotic characteristics estimated in case of biological signals seem to be relative and why they cannot mirror the properties of the hypothetical models. In our opinion, the length of an analysed time interval is strongly dependent on the kind of brain processing, and, in the case of spontaneous ("uncontrolled") brain activity where uncontrolled task changes occur, lies anywhere in the range of $0.5 \mathrm{~s}$ and a minute or so.

\section{The Chaoticity}

For nonstationary systems, the total system energy is not constant across time. This means that there is a change of energy and entropy for two subsequent time segments. Scanning the measured signal with the algorithms for nonlinear measures in form of LLE or K-S entropy will characterize the stationarity of the measured signal (at the correct choice of the time segment). In general, there are time series in which the average change is rapid and other time series for which the average change is gradual. Rapid change indicates phase-transition like behavior [Aizawa, 1986; Badii, 1988; Fuchs \& Kelso, 1992]. In order to observe phase transitions one needs a well defined order parameter. Experimentally, this means that we need at least one control parameter (such as temperature or an electric field, etc.). This has not been realized for spontaneous activity measured in EEG/MEG experiments. Nevertheless, we expect to observe rapid changes of the signal because different processes in the brain are connected with different kinds of brain dynamics which are limited in time. Let us now assume that if different processes correspond to two different attractors, then the switching between these attractors constitutes a critical transition. An EEG or MEG signal recorded under resting conditions is usually both nonstationary and high-dimensional. Adding of an uncorrelated process to the other ongoing processes in the brain will increase a number of degrees of freedom. Imagine, for instance, a model of brain activity such that an external event could trap the system for a brief period in any one particular attractor. There will be attracting transient trajectories. The appearance of the attractor, however, will be too short, such that only a fuzzy image can be reconstructed. There will not be enough orbits to search in many dimensions; con- sequently, it will not be possible to estimate the traditional chaotic features. The scanning procedure which we present provides a possible solution to this problem.

A sudden change in the system dynamics does not necessarily mean a loss of deterministicity. A change in attractor properties, i.e., in the type of motion, can occur in well defined systems such as in the case of an interior crisis [Grebogi et al., 1982, 1983; Lai et al., 1992; Franaszek \& Nabagło, 1993]. We have experimentally observed such critical jumps in the bouncing ball [Kowalik et al., 1988]. In this case, the attractor consists of two topologically different parts, a periodic or quasiperiodic region of small jumps, and another randomly reanimating "strange" part. The ubiquitous noise caused alterations in the system dynamics. The situation for EEG/MEG may be comparable, i.e., the informational external noise could evoke the change of the brain-attractor.

As already mentioned, the LLE may be useful in evaluating chaoticity if the investigated behavior is stationary and deterministic. However, the problem of detecting and quantifying chaoticity has not yet been solved. Our goal here was to develop a chaoticity measure based on the definition of the Lyapunov exponent, which allows conclusions even when constraints regarding stationarity cannot be met and which, consequently, can be utilized for the analysis of most experimental data sets. Moreover, it should be applicable to a system with an unknown number of degrees of freedom (this can be derived for low-dimensional and stationary systems by estimating the fractal dimension up to the saturation level). The greatest problem when estimating the LLE from experimental data is an insufficient amount of data and the sensitivity of the estimation to noise. The first problem is simply a problem of nonstationarity, because of the dependence of energy on time ( $\propto$ number of points). Because the variable in Eq. (5). has to be timedependent in such a case, our proposal is to use averaging after time [in addition to the small ensemble averaging as in Eq. (5).] Therefore, we can define chaoticity $\Lambda$ as:

$$
\Lambda=\langle L(\alpha, t)\rangle_{\Delta t}
$$

where $\Delta t$ denotes averaging across a time-interval (scanning window), $\alpha$ is a symbolic notation for all parameters used for the reconstruction of the attractor, and $L$ is estimated from Eq. (5), for fixed $i$ (only one arbitrary reference trajectory). 
The next problem is the noise level. The slightest change in the digital representation of data can result in a different $L$-estimate. In order to avoid this problem we propose to base the computations on a normalized data set or to use directly the integer data produced by $\mathrm{A} / \mathrm{D}$-conversion. The reasoning for this choice is that the thickness of the trajectory in the best case will be equal to $1 \mathrm{bit}$ and numerical noise will be at least equal to 2 ( \pm 1 bit).

Let us assume a time series generated by a multidimensional system with an unknown dimension which is not long enough to obtain a satisfying estimate of the correlation dimension (no saturation with growing embedding dimension). Consequently, it will also be more difficult to estimate the correct value of the LLE as the reconstruction of the attractor cannot be sufficiently detailed [Eckmann \& Ruelle, 1992] and, hence, the LLE will not converge with an increasing number of points [Wolf et al., 1985]. Nevertheless, we may obtain convergence with the number of iterations. In this case, the value reached will obviously not be the

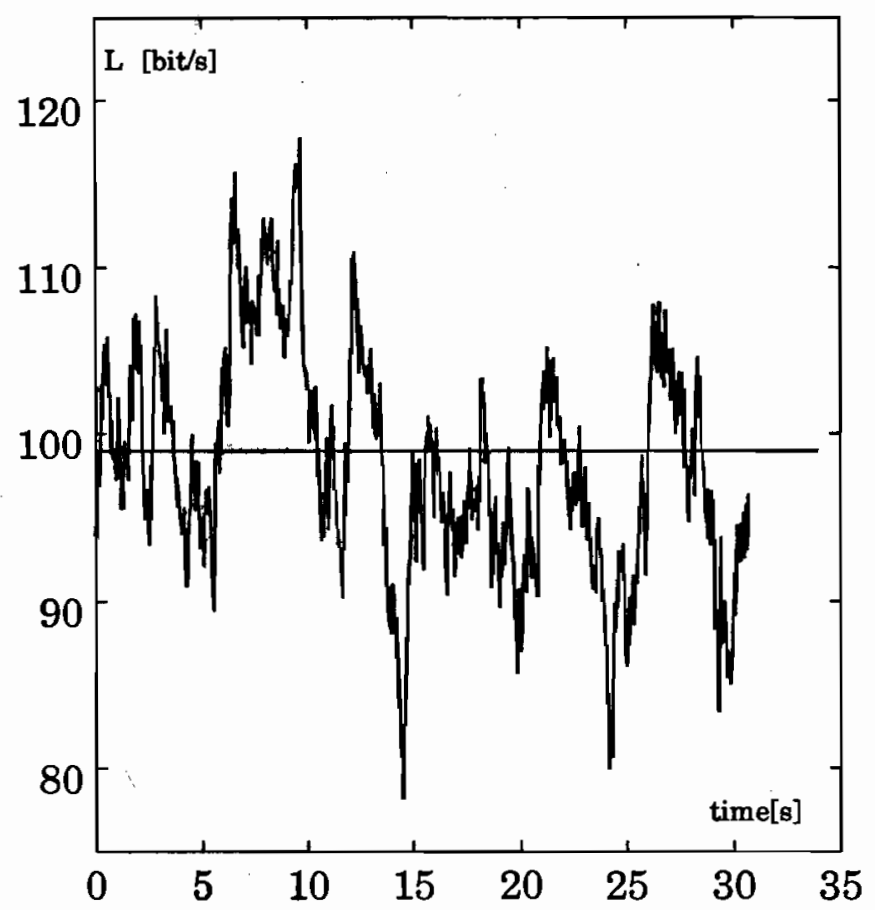

Fig. 1. A scanning of the largest Lyapunov exponent in Henon map by use of Wolf's algorithm [Wolf et al., 1985]. 8192 points of Henon attractor (for $a=1.4 ; b=0.3$ ) were calculated, and the estimations of LLE were performed in a window consisting of 512 points, the window shifted consecutively by 5 points. Embedding dimension $e=4 ; d t=0.005$; $\tau=1 ;$ and evolv $=1$. correct LLE. Consequently, a different choice of time slices will result in different values of the estimated limits. It is independent of the particular algorithm applied. Figure 1 presents the changes of the LLE estimated using the method of Wolf et al. [1985] for scanned time series with a scanning window consisting of 512 points and shifted about 5 points for the consecutive calculation.

As can be seen, the LLE oscillates quite randomly with an average level of 99 . This value will be closer to the true value of 106 for a larger number of data points (for 2048 points in the window the oscillations have much smaller amplitude and the average level is lying about 106). Generally, this statement is valid on a statistical basis only. This is particularly true as the values may oscillate with number of iterations before saturation is reached. In spite of such systematic error caused by small amount of data points, it seems to be possible to use these underestimated (for more dimensional systems - overestimated) values for the comparative studies. A calculation of the LLE by use of the scanning technique is very time consuming if the methods like Wolf et al. [1985] are applied. Therefore, we have directly applied the definition of local Lyapunov exponents to our method [Eqs. (3), (4)]. We propose the following procedure: Let us first assume we have a deterministic signal measured in an experiment and measured with an accuracy of $B$-bits. Before we start tracing two closeby trajectories we scale a whole data set to the integer range $\left[0 \ldots N \mathrm{MAX}=2^{B}\right]$. Now let us choose a time slice. (Even if that slice is too short to estimate a proper LLE value, it can still be compared with a corresponding value estimated for the next slice.) For a fixed embedding dimension, we then follow nearby trajectories and estimate the rate of their average exponential divergence according to the definition of local exponent. Following this step, we shift the chosen time sequence around the few points and repeat the procedure. After $N$ scan steps, we obtain $N$ different values of quasi-LLE. What we now have is a new time series of the quasi-LLE's which allows us to characterize the momentary stability of the system.

\subsection{An algorithm for estimation of the chaoticity}

Summarizing the above description, we need to perform the following steps in order to estimate the chaoticity: 
(i) Read the block of data - usually 512-8192 points.

(ii) Normalize the block (typically a 12 bit A/D converter, i.e., the range $[0,4095])$. This step is required because we are looking for "pure" system dynamics changes and the amplitude relations for the successive scanned windows are not important. This is also the moment where eventual artifacts in the signal have to be eliminated, as they can momentarily change the scale of the observed dynamics.

(iii) Choose an initial point (arbitrarily choose one of the initial points which is larger than the delay) and the parameters for phase space reconstruction (embedding dimension, delay, step). This choice can be performed by identifying the first zero-crossing of the autocorrelation function [Graf \& Elbert, 1889].

(iv) Find all neighbors which are closer than arbitary minimal distance. The smallest distance must be greater than noise level (as mentioned noise level $\geq 2$ bit).

(v) As a rule, if the distance is equal to zero in estimations we exchange the zero with one except of the starting distance that will be exchanged by $\operatorname{dist}(O)=\mathrm{NMAX}^{2}$. This has a strong implication in the behavior of quasiLLE: no zero means no $-\infty$ of the exponent. Due to noise there is no possibility of detecting zero-distance in the real data and the above method will probably be more realistic in an experiment. However, because of the mentioned replacements, the result will be a positive exponent instead of a negative exponent in the case of periodic motion. This means that the Lyapunov's definition of such an exponent is no longer valid for the periodic (or transitory periodic, quasiperiodic) orbits. Nevertheless, as will be seen from our examples, the measure will more than adequately describe qualitative state changes.

(vi) Use the definition of the local Lyapunov exponent given in Eqs. (3)-(5).

(vii) Average within the scanning window [Eq. (8)]. This point can be performed after the whole scanning procedure and the window width applied can differ from the scanning window. We prefer the last method. Usually for a scanning window of 512 points an averaging within a window of $64-512$ points in width is performed. (viii) In the outcome, we observe local changes of the LLE as well as the changes of the average $\Lambda$ (after $\Delta t$ ) and its variance $\sigma_{\Lambda}$. As we will see in further examples, the chaoticity of the measured signal and its changes can be described by all three measures $\left(L, \Lambda, \sigma_{\Lambda}\right)$.

Figure 2 presents an evolution of the static values of the chaoticity for the logistic map as a function of the nonlinear parameter.

The changes of the chaoticity presented in Fig. 2. are more similar in form to those observed for the entropy than to those in the evolution of the Lyapunov exponent. Moreover, as shown in Fig. 2(b), the values of chaoticity are proportional to the number of bits, and, for instance, for 12-bit converting these can be from -12 to 12 ; in general, for $\beta$-bit converters they reach values from the region $[-\beta,+\beta]$.

Figure 2(c) illustrates the influence of the normalization, i.e., step (ii) of the procedure. Another example can be a synthetically assembled signal formed by intervals of Lorenz, Duffing, and Vander-Pol-like chaotic attractors interrupted by the combined oscillation (sum of three incommensurate sine functions with frequency ratio 31:13:7). Figure 3 shows the corresponding evolution of the chaoticity for this time series.

For the estimations presented in Fig. 3 we have used the same fixed set of parameters (embedding dimension, delay, step, scale).

It is very important to point out that we have also observed sudden changes in the deterministic and stationary models. Such transitions can be found in many nonlinear systems, among them being the Van-der-Pol-like oscillator [Kowalik et al, 1995a]. In this oscillator, a series of transitions between different types of dynamics takes place.

Figure 4 shows an example of a very distinct change in the dynamics of the Van-der-Pol-like oscillator. The presented time slice contains 13000 points [Fig. 4(a)] and exhibits, almost in the middle of the plot, a sudden change in its dynamics. This point is depicted by a small arrow, close to 6200 in Fig. $4(\mathrm{a})$. The attractor belonging to the whole slice is shown in Fig. 4(b), and those corresponding to the left and right hand side regions are presented in Fig. 4(c) and 4(d), respectively. Figures 4(e) and 4(f) show the scanned changes of the chaoticities $\Lambda$ and $\sigma_{\Lambda}$; The rapid change of the chaoticities levels which take place very far before the transition 


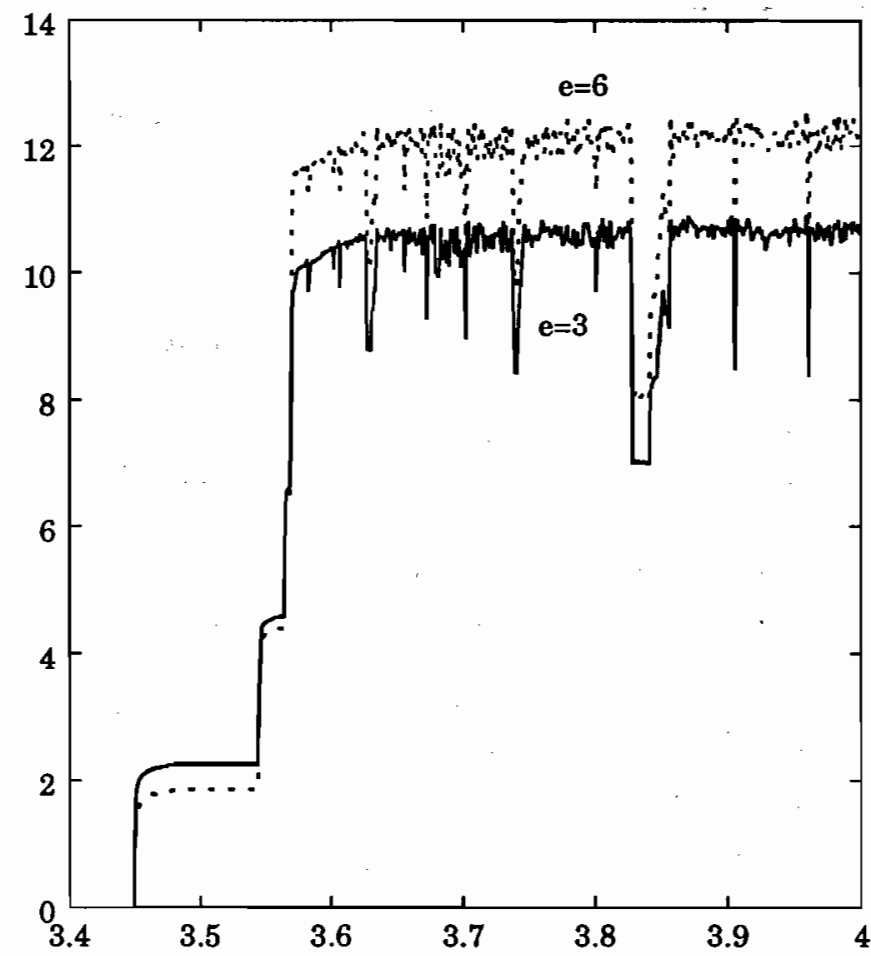

(a)

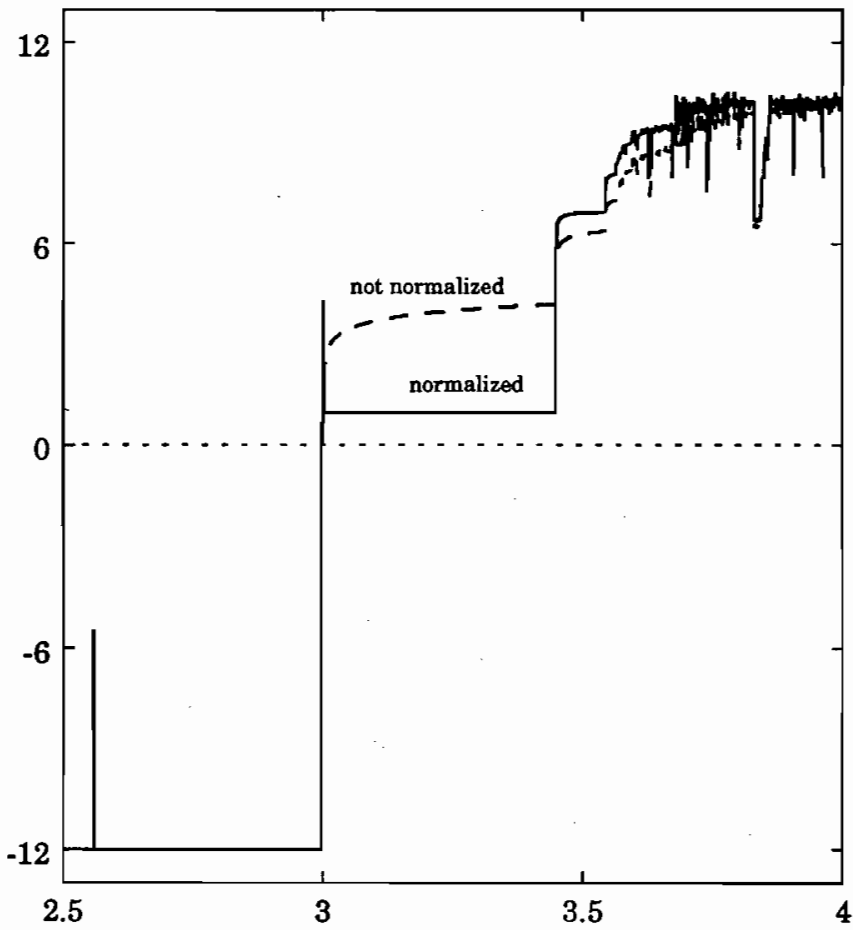

(c)

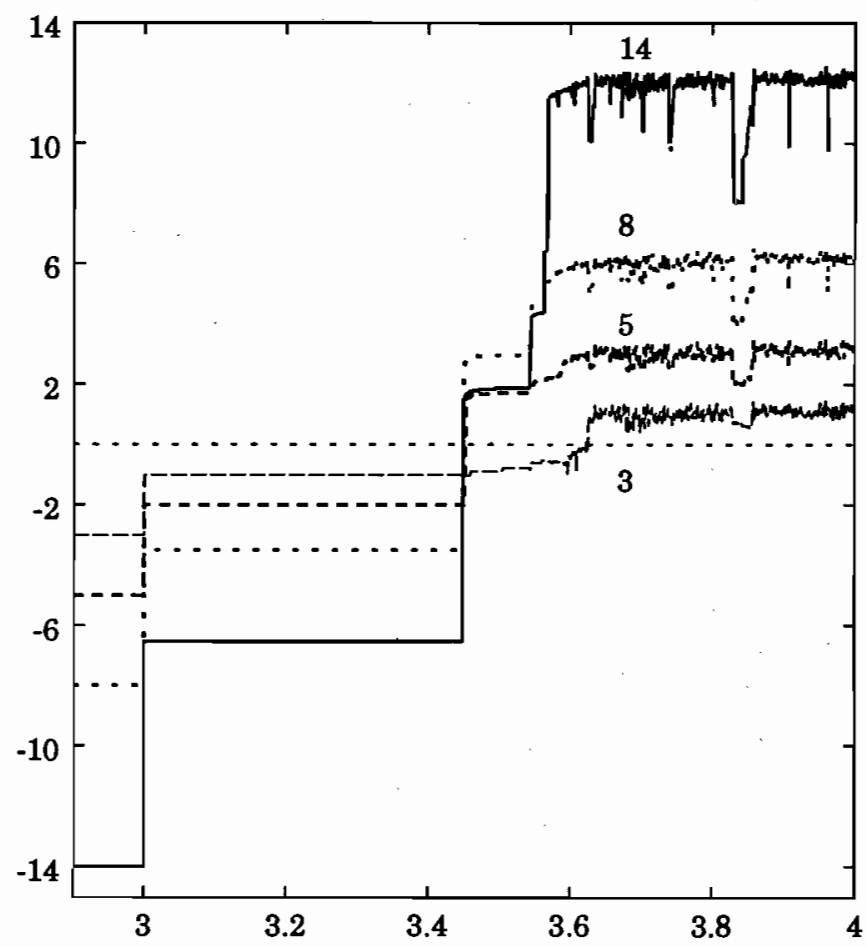

(b)

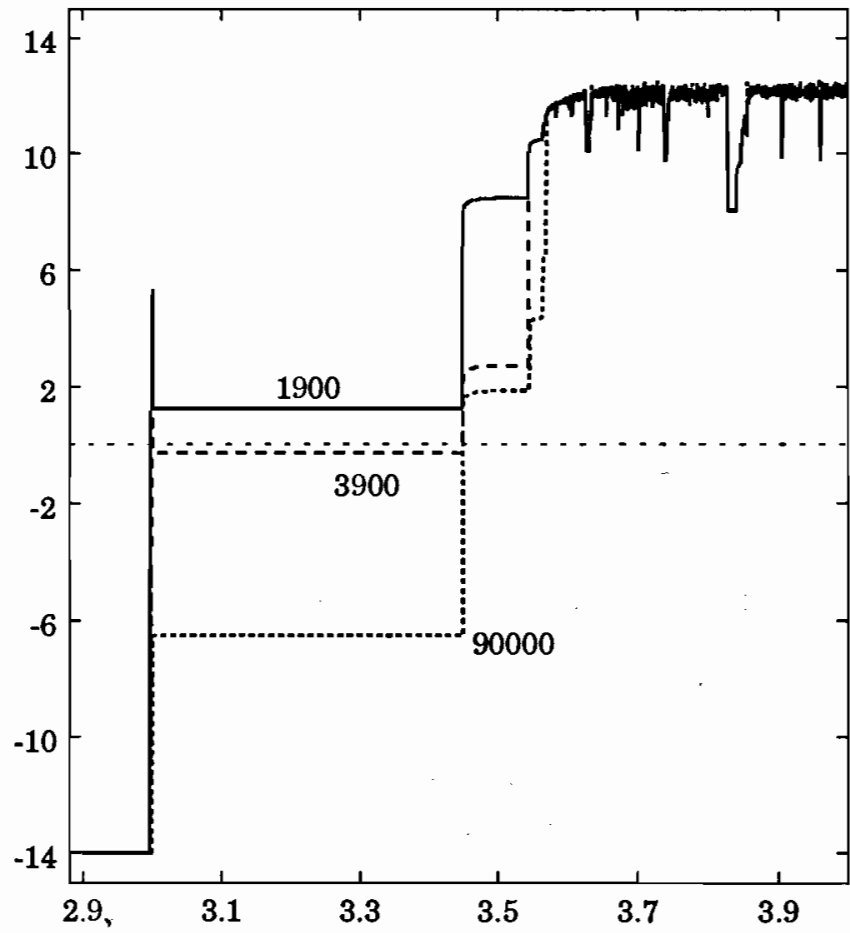

(d)

Fig. 2. Exemplifying the chaoticity on a logistic map. (a) Dependence of chaoticity on an embedding dimension and (b) on accurracy of digital presentation. (c) Influence of the block normalization procedure on the evolution of chaoticity. As seen for the unnormalized blocks, the superstable point is also visible and there are no sharp changes between states. (d) The sensitivity of the chaoticity measure is shown by the evolution of the chaoticity as a function of number of initial iterations (Note: There are almost no differences in the chaotic region). 


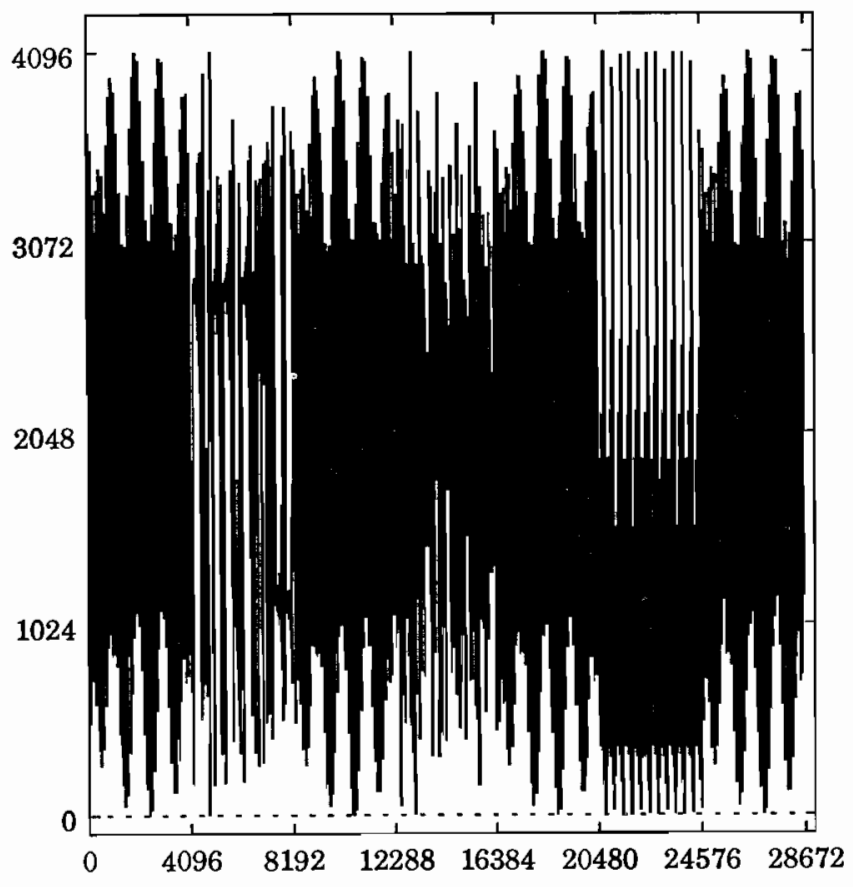

(a)

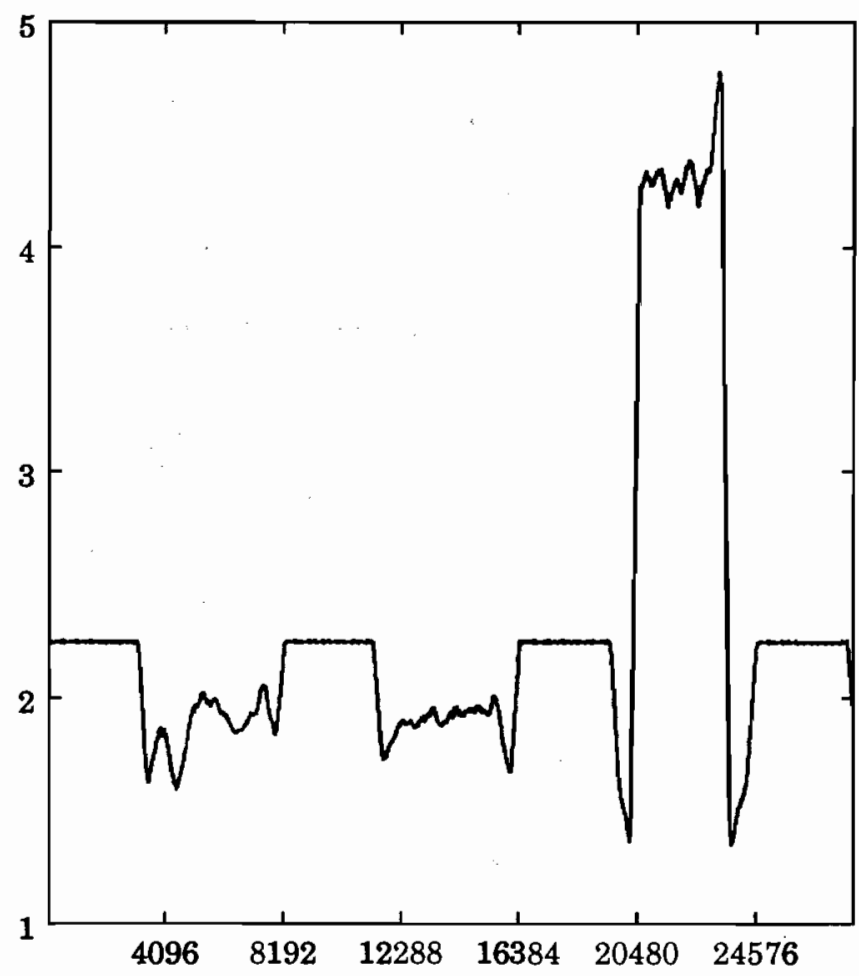

(b)

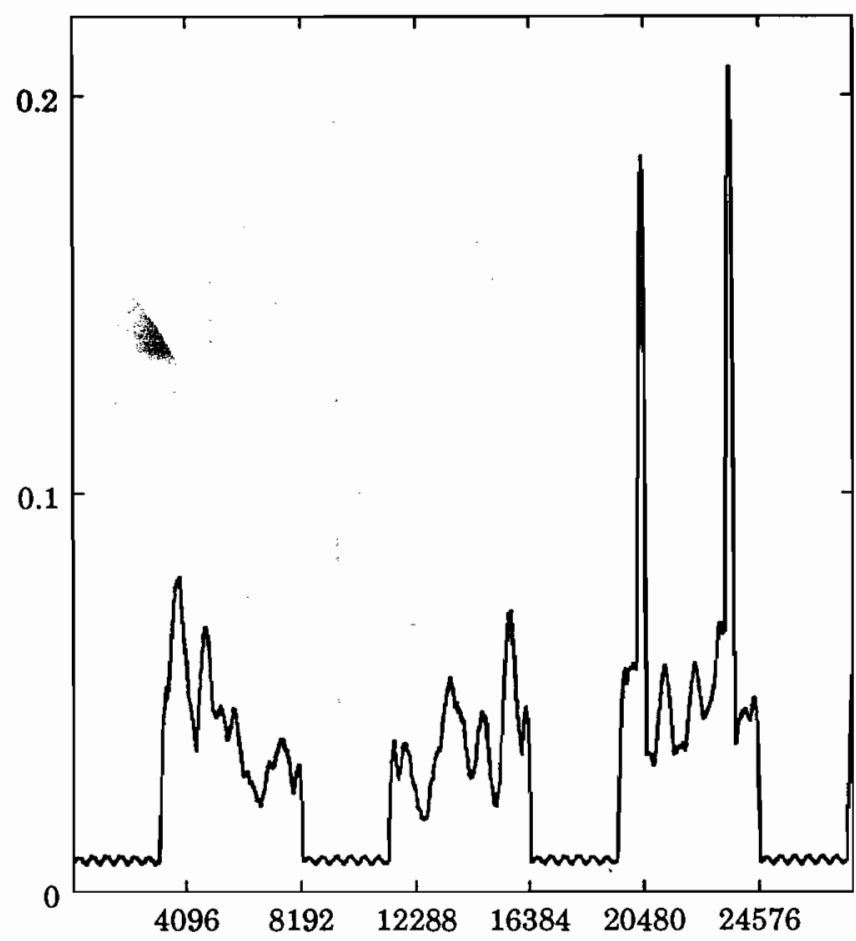

(c)

Fig. 3. (a) A synthetized time series containing 7 intervals, each 4096 points. These are successively: sine combination: $\cos 2 \pi f_{1} t+\sin 2 \pi f_{2} t+\sin 2 \pi f_{3} t$ (with $f_{1}: f_{2}: f_{3}=31: 13: 7$ ), Lorenz attractor, sine again, Duffing, sine, Van-der-Pol-like, sine. (b) Its chaoticity $\Lambda$ and (c) Chaoticity $\sigma_{\Lambda}$.

The variability $\sigma_{\Lambda}$ shows more distinctly the changes of the system dynamics. Embedding dimension $e=5$ and delay $\tau=40$ were used. 


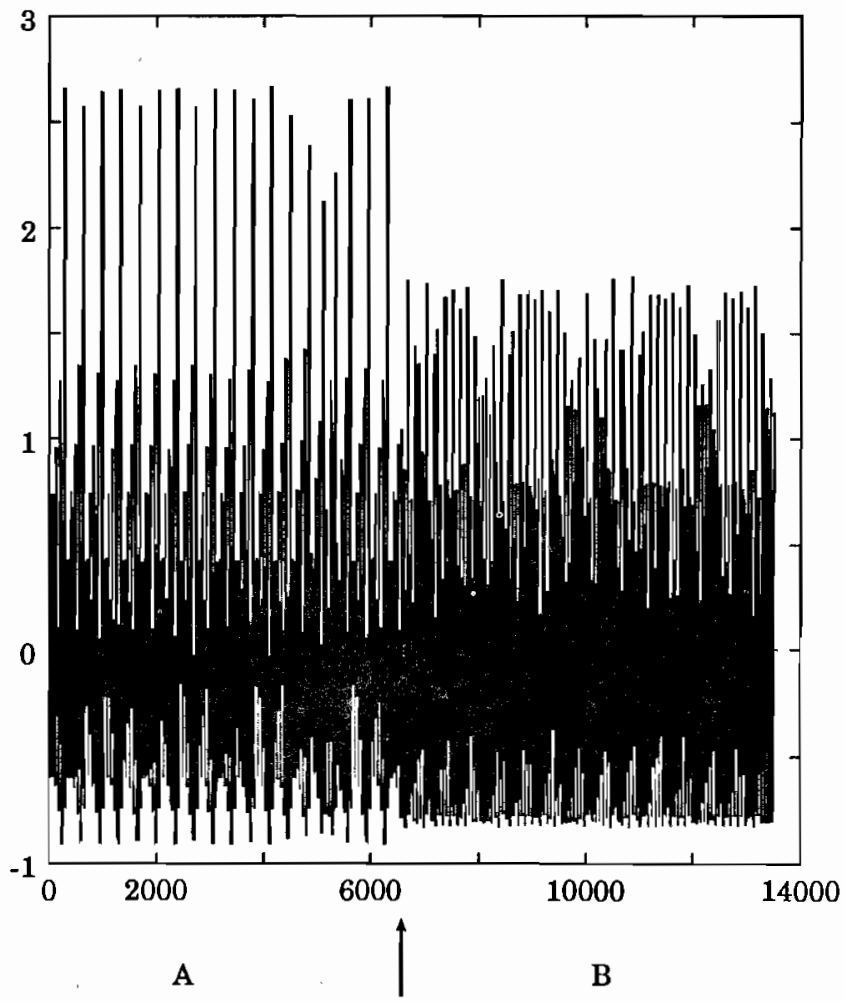

(a)

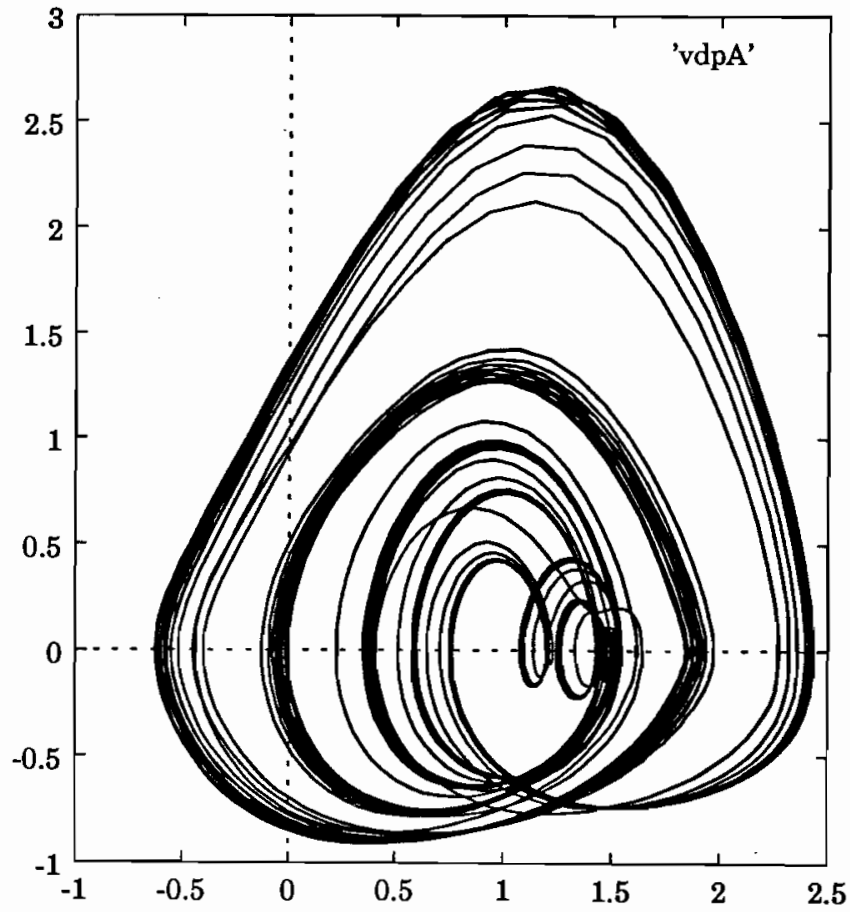

(c)

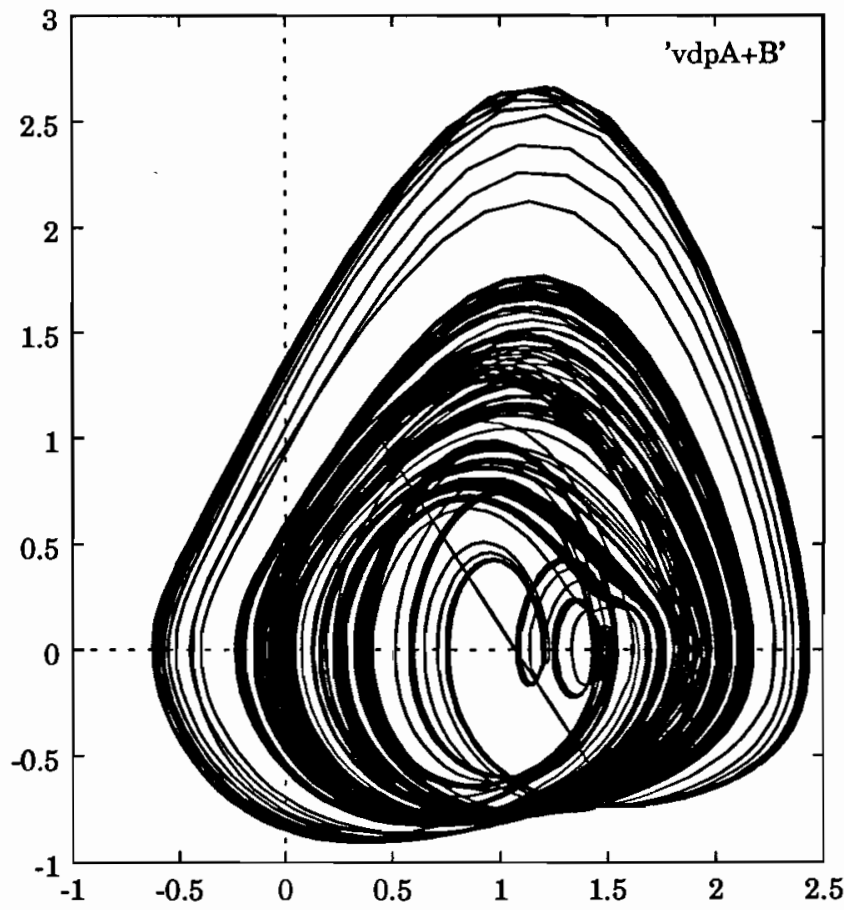

(b)

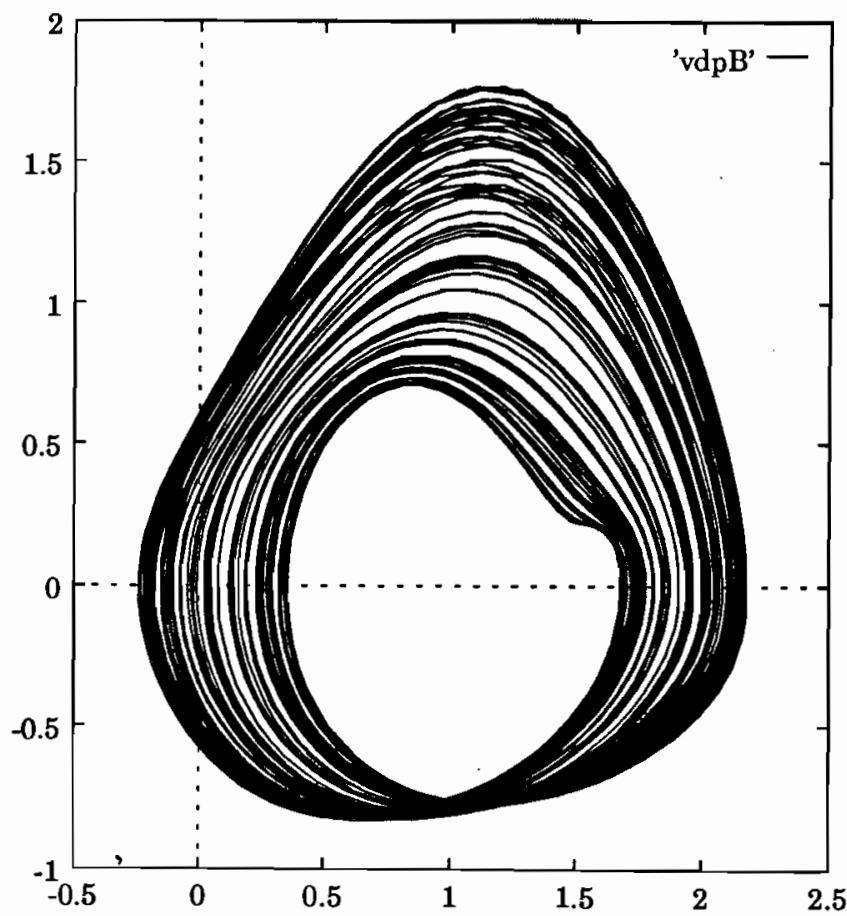

(d)

Fig. 4. Sudden changes in the Van-der-Pol-like oscillator [Kowalik et al., 1995a]. (a) A time series consisting of 13512 points; (b) A global attractor; (c) and (d) The partial attractors of the left and right side, respectively; (e) The evolution of chaoticity $\Lambda$; (f) The same for $\sigma_{\Lambda}$; (g) The scanned Wolf's et al. [1985] LLE. The VDP-like oscillator is governed by the equation: $\ddot{X}=-\dot{X}\left(\frac{c}{a}+a(1-\Lambda \cos X)\right)-X-c(X-\Lambda \sin X)+c\left(Y_{o}+Y_{1} \sin 2 \pi F \tau\right)$. The presented signal was derived for $F=0.288$, $c=0.25, a=0.8, Y_{o}=3.1, Y_{1}=2.45, \Lambda=3.0$. 


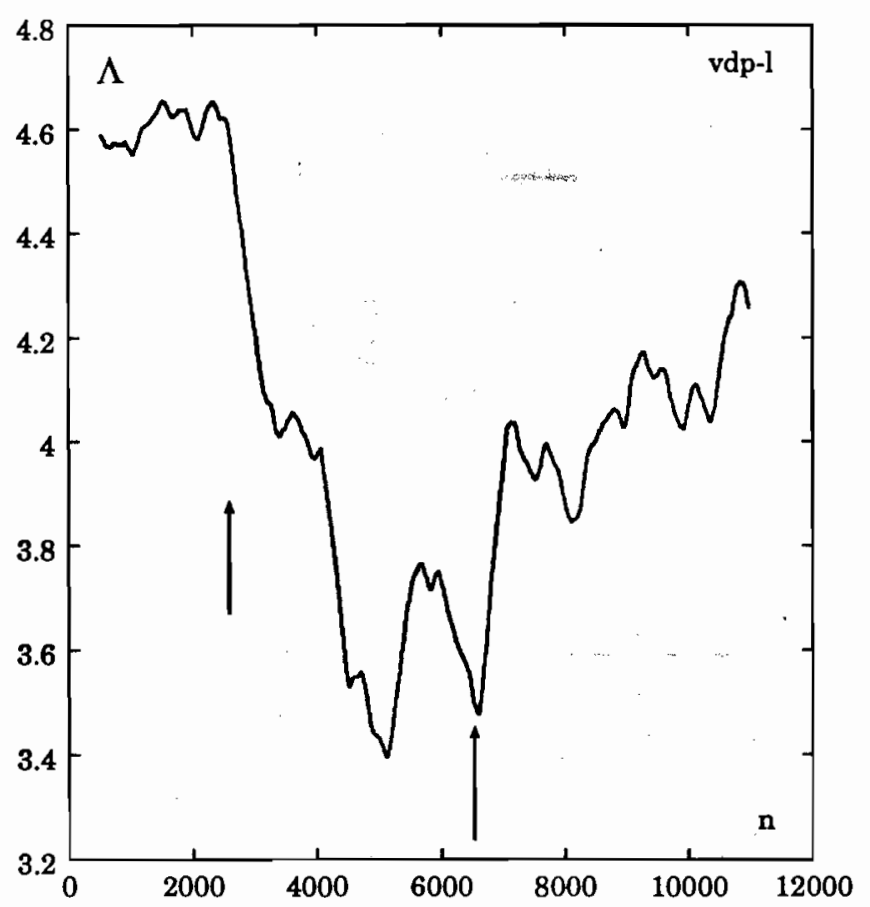

(e)

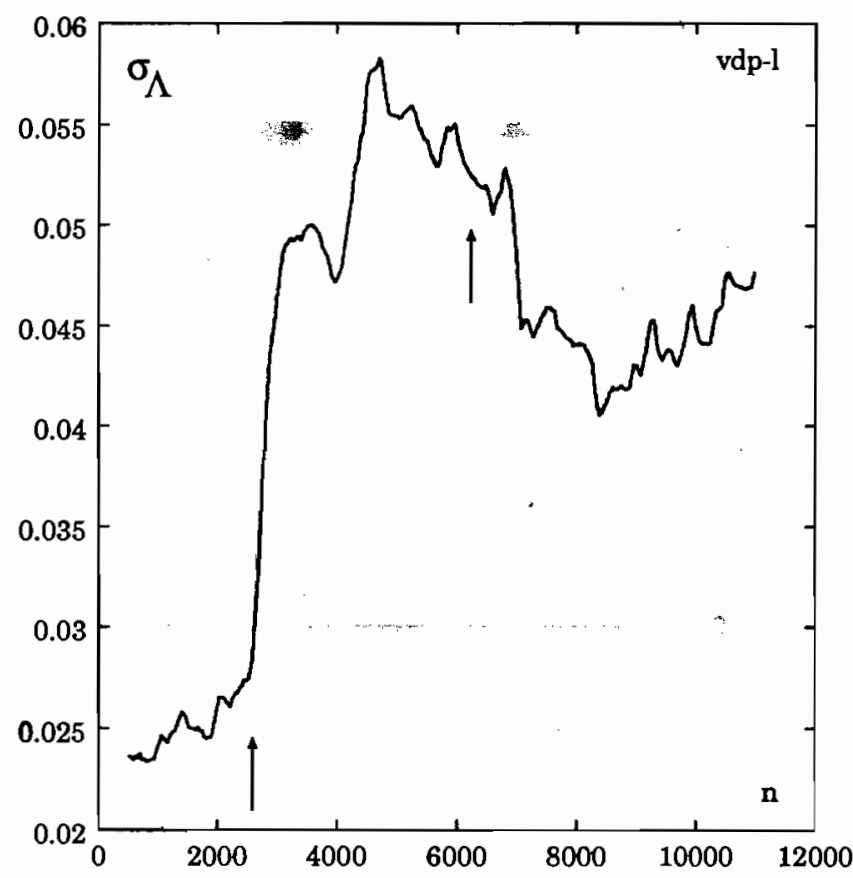

(f)

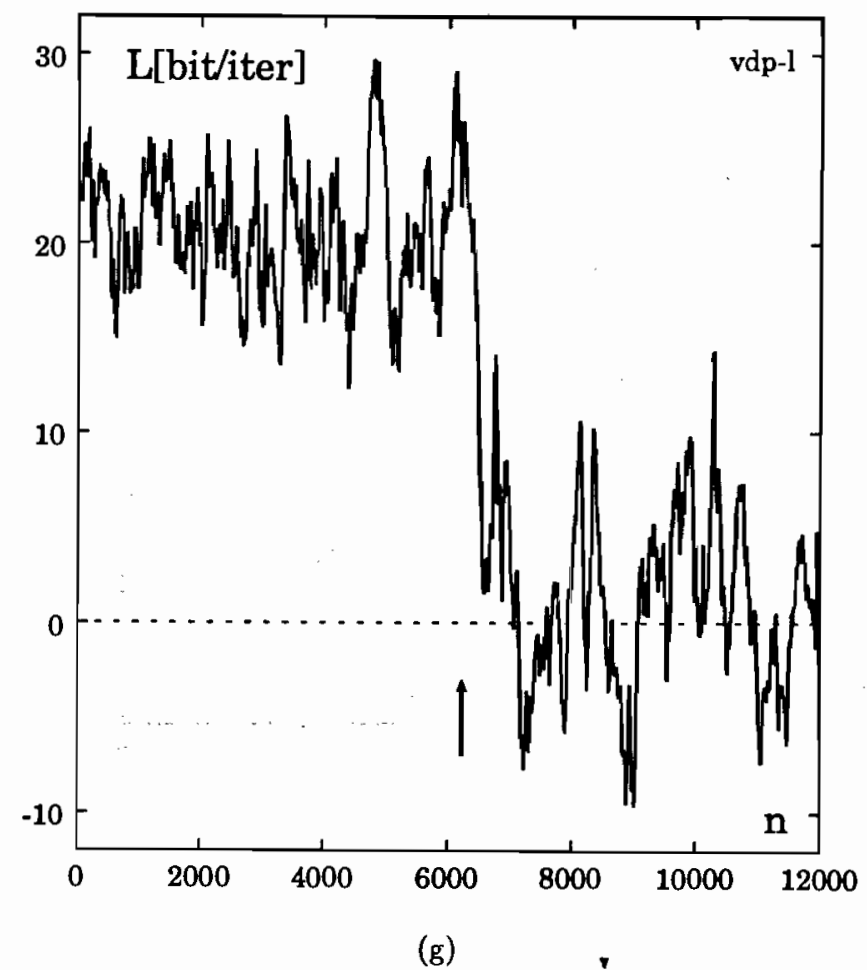

Fig. 4. (Continued)

point is visible at this point. It is not an effect of windowing because the window's width is 512 points in this case and the precursor of transition is around the point 2500 . On the other hand, the jump of the "classic" LLE in Fig. 4(g) is situated exactly in the point of transition. As a precursor, a growing variability of Lyapunov exponent beginning at 3000 can be observed. An even more interesting 


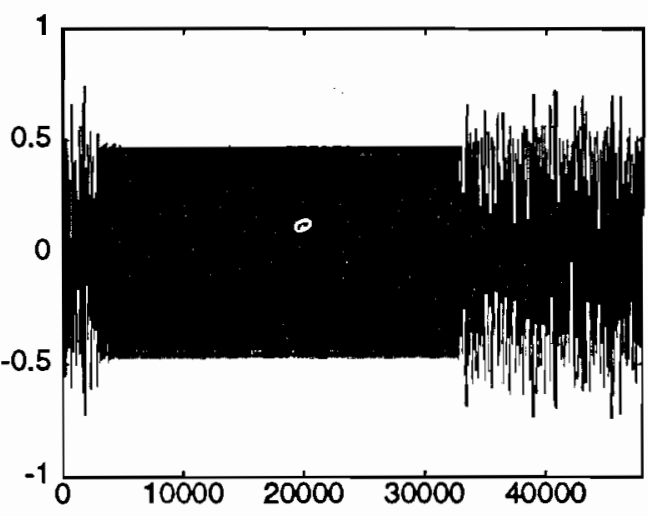

(a)

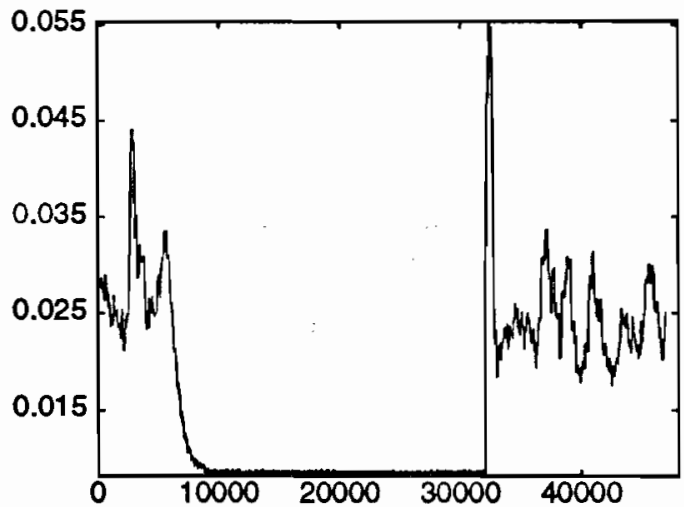

(c)

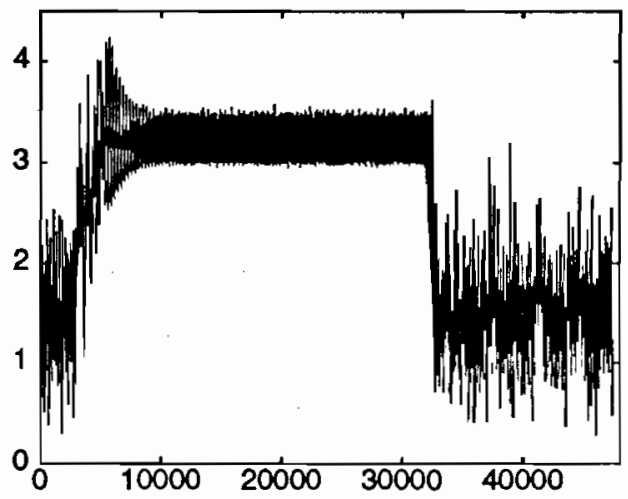

(b)

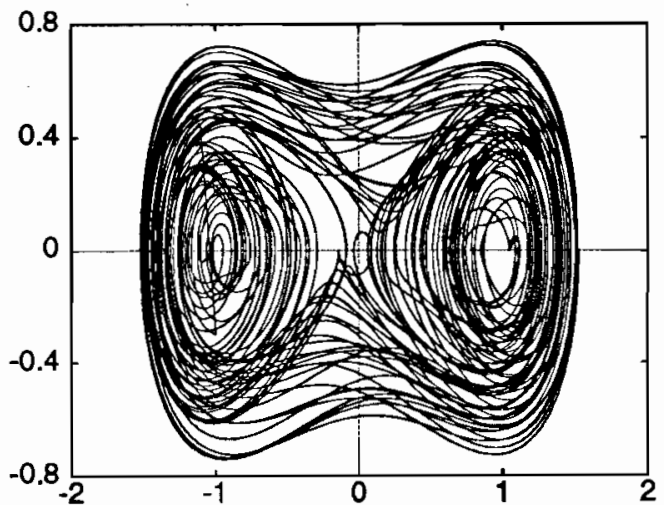

(d)

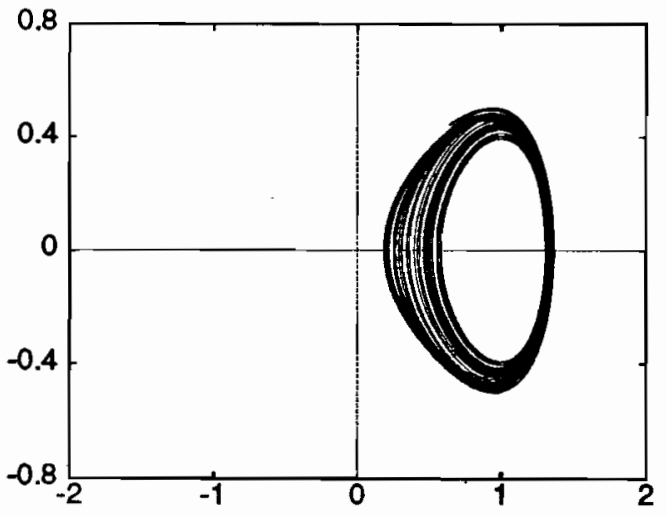

(e)

Fig. 5. Crisis-induced intermittency in a Duffing oscillator [Franaszek \& Nabagło, 1993]. (a) Time evolutions of $\Lambda$ (b) and $\sigma_{\Lambda}(\mathrm{c})$ show a jump between quasiperiodic and chaotic parts of the attractor. This jump corresponds to a change of the form of the attractor from double-wings (d) to single-wing quasiperiodic cycleș (e).

example is a two-wing attractor which represents a Duffing oscillator. With a particular combination of parameters, there exists an intermittent crisis which is easily observed [Franaszek \& Nabagło, 1993]. The signal in this regime and changes of values $\mathrm{L}, \Lambda$, and $\sigma_{\Lambda}$ are plotted in Fig. 5 .

Since we assumed in our estimations that the reconstructed part of the attractor in each of the 
time slices results from varying dynamics, the respective data set of particular window was independently normalized. The advantage of such normalizing is a weak dependence of the measured value on the artifacts and a better description of the changes in system dynamics. The average values estimated in the method proposed above and the corresponding variance will henceforth be referred to as the chaoticity measures.

\section{Possible Applications to MEG/EEG Time Series}

As we have seen from the previous examples, there is no temporal change in the average of the quasiLLE series for nonlinear stationary oscillations if no rapid change of system dynamics occur. This is represented in the following experiments:

(i) The chaoticity can be used for mapping functional brain activity similar to the LLE [Kowalik et al., 1993].

(ii) For the magnetic fields and electric potentials produced by the human brain we can observe sudden changes of the system dynamics.

(iii) The changes of the chaoticity measures takes place earlier than the visible change of the attractor structure; thus, they can be used as transition predictors.

The evaluation of dynamical patterns relies on the analyses of spontaneous brain activity, where the signal is generated without a specially defined task. The data analysed were the results of two investigations: One where MEG was measured in tinnitus (continuous ringing in the ear) patients and a second where EEG time slices were collected from schizophrenia patients.

For the numerical analysis, only artifact free intervals containing more than 10000 points were used. (For EEG it was at least $100 \mathrm{~s}$ for $100 \mathrm{~Hz}$ sampling rate, bandwidth $\mathrm{DC}-100 \mathrm{~Hz}$, and for MEG it was more than $10 \mathrm{~s}$, sampling rate $862 \mathrm{~Hz}$, bandwidth DC-200 Hz.) The (central) EEG referred to linked mastoids was recorded in cooperative schizophrenic patients during a 20 -minute interview with their therapist. The EEG was simultaneously recorded from the interviewer.

MEG-signals were measured using a 37 Channel magnetometer (BTi, San Diego, USA) consisting of 37 DC-SQUIDs operating at liquid helium temperature. The magnetic measurements were performed inside a magnetically shielded room to reduce environmental noise. The dewar containing the magnetometer was centered over the temporal lobes of both hemispheres successively. Spontaneous activity was recorded for 18 subjects under resting condition. For the measured data set we have estimated the spatial distribution of the chaoticity. Figure 6 presents one representative plot of a chaoticity-map measured over the left hemisphere.

Distinct local variations suggest that under resting conditions the brain dynamics vary considerably between regions, meaning that there is only limited or even no coupling among the different brain regions. On the other hand, nearby channels often display equal values in their dynamical variables. This cannot be explained on the basis of magnetic field propagation alone. The nonhomogeneous image of the chaoticity maps (the same

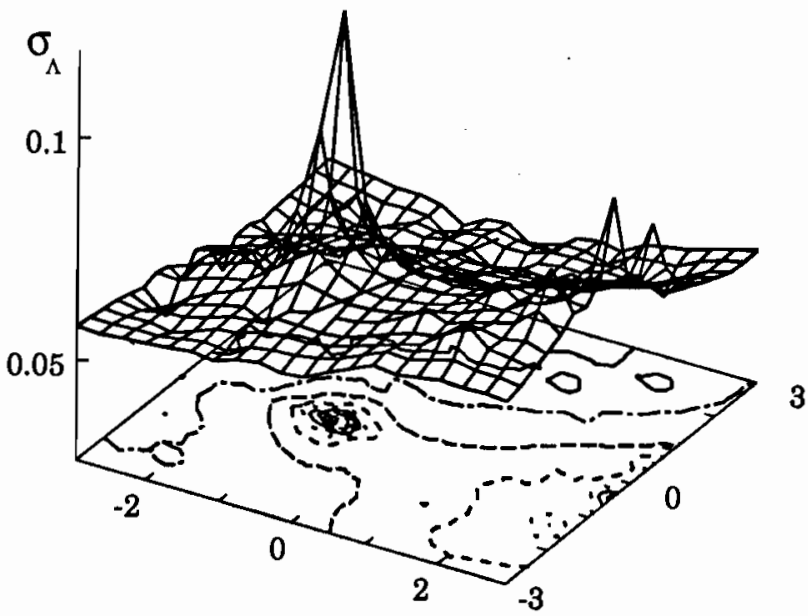

Fig. 6. Spatial distribution of chaoticity $\Lambda$ derived from human MEG. 


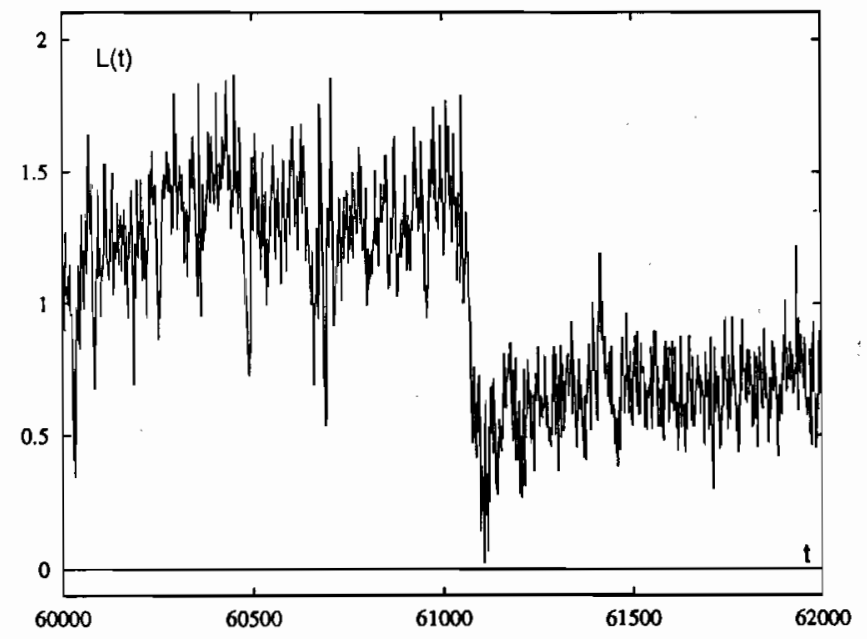

(a)

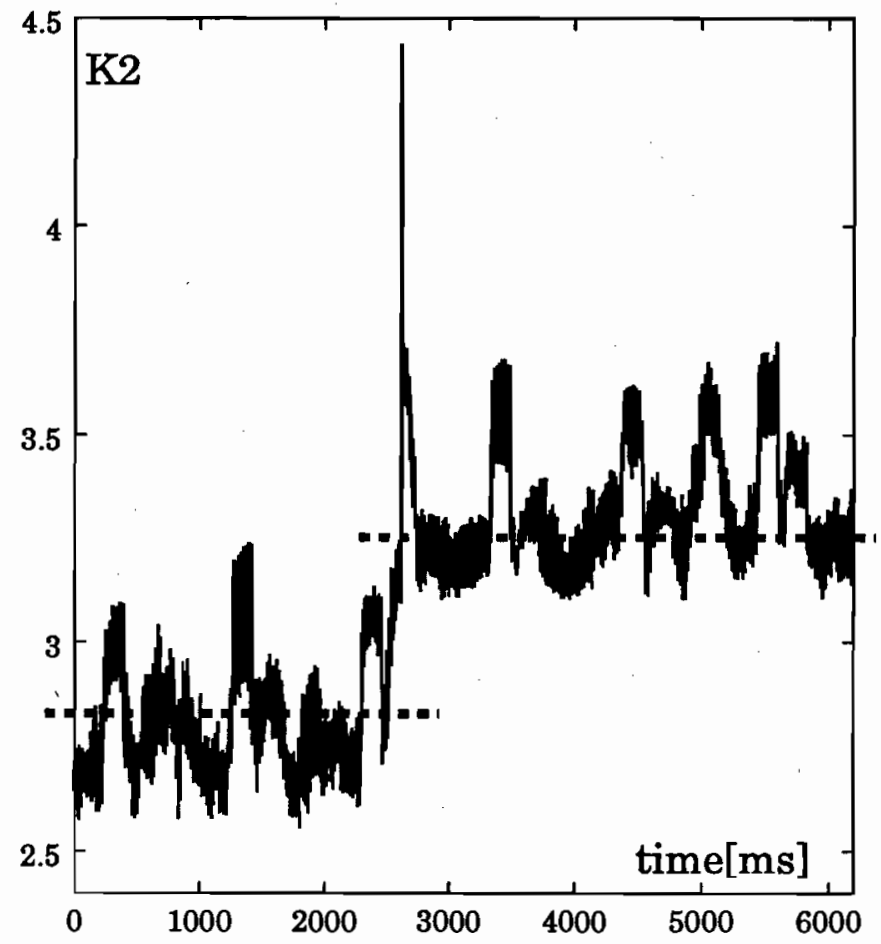

(b)

Fig. 7. Sudden changes of brain dynamics can be detected both by the estimation of chaoticity and entropy. Rapid change of chaoticity in the EEG is shown in plot (a) and a change of entropy for an MEG series is shown in (b). Not shown here: The changes of entropy for EEG and the chaoticity of MEG are similar in character.

observed for LLE [Kowalik et al., 1993]) indicates the task-dependent coupling of various brain regions.

The change of the average value is very rapid and the range of that change significant. There is convincing evidence that the use of the chaoticity measure can describe the delicate and invisible changes of the system dynamics across time.

Of course, we cannot expect that the dynamical states and the attractors which characterize such states remain constant across time. If the attention switches to another task we may expect variations in both the dynamical characteristics and the coupling between brain areas. The time evolution of the chaoticity suggests that this is indeed the case (Fig. 7).

The visible jump in the middle part of the plot can be viewed as a phase transition-like phenomenon, i.e., a transition in the dynamical flow of the brain activity. Such critical transitions are exhibited significantly more frequently in patients with a paranoid-hallucinatory disorder than in the interviewer, for whom EEG was simultaneously being recorded. Similarly to this presented in
Fig. 7(a), for a critical jump of chaoticity for the EEG time series one can observe such a jump also for MEG-signals (in Fig. 7(b) presented as the jump of entropy).

\section{Final Conclusions}

In order to uncover the mechanisms underlying neural mass activity it is not sufficient to analyze the amplitude and/or frequencies of the measured MEG/EEG signals. In keeping with brain activity, it seems necessary to analyze the dynamical patterns, i.e., to follow the temporal changes (for example, the informational capacity) of the largest Lyapunov exponent [Kowalik et al., 1993]. The presently introduced method involves the estimation of the chaoticity of a signal with several advantages:

- all types of experimental data can be analyzed

- computer estimation is quick

- the method can also be applied to a short time series 
- it supplies the same information about the system dynamics as the entropy rates and LLE [Iasemidis \& Sackelares, 1991;Kowalik et al., 1993; Elbert et al., 1994; Kumpf et al., 1995].

Moreover, it enables a direct (i.e., on-line) measurement of the chaoticity. Despite the problems of interpreting this measure for cases of periodic or quasiperiodic motion, it can very sensitively indicate such states. As the average change of chaoticity estimated from the EEG/MEG signals characterize the temporal information flow in the brain, clinical applications are within reach. Therefore, it seems worthwhile to test whether the information extracted will prove to be of diagnostic value in the clinical practice.

Finally, we should mention that the proposed procedure may be useful for the analysis of a variety of biosignals for the analysis of data from other scientific disciplines. One such example is the analysis of behavioral data being presented in Schiepek et al. [1995] and Kowalik et al. [1995b].

\section{Acknowledgments}

The authors would like to thank Brigitte Rockstroh and Hans Watzl for providing EEG data. Special thanks to Bernd Lütkenhöner for very useful remarks and to Scott Hampson for stimulating discussion. We also thank Christina Robert for her help in the preparation of this manuscript. Research was supported by the Deutsche Forschungsgemeinschaft (Ro805/4-1).

\section{References}

Aizawa, Y. [1986] "Chaos-chaos phase transition and dimension fluctuation," in Dimensions and Entropies in Chaotic Systems, ed. Mayer-Kress, G., Springer Series in Synergetics, Vol. 39 (Springer-Verlag, Berlin, Heidelberg, New York, Tokyo), pp. 34-41.

Babloyantz, A. [1985] "Strange attractors in the dynamics of brain activity," in Complex Systems-Operational Approaches in Neurobiology, Physics and Computers, ed. Haken, H. (Springer, Berlin), pp. 116-122.

Badii, R. [1988] "Generalized thermodynamic ensembles for fratal measures," Il Nuovo Cimento 10, 819-840.

Brown, R., Bryant, P. \& Abarbanel, H. D. I. [1991] "Computing the Lyapunov spectrum of a dynamical system from observed time series" Phys. Rev. A43, 2787-2806.

Buzug, Th., Reimers, T. \& Pfister, G. [1990] "Optimal reconstruction of strange attractors from purely geometrical arguments," Europhys. Lett. 13, 605-610.
Destexhe, A., Sepulchre, J. A. \& Babloyantz, A. [1988] "A comparative study of the experimental quantification of deterministic chaos," Phys. Lett. A132, 101-106.

Eckmann, J. P., Kamphorst, S. O., Ruelle, D. \& Ciliberto, S. [1986] "Lyapunov exponents from time series," Phys. Rev. A34, 4971-4979.

Eckmann, J. P. \& Ruelle, D. [1992] "Fundamental limitations for estimating dimensions and Lyapunov exponents in dynamical systems," Physica D56, 185-187.

Elbert, T., Ray, W. J., Kowalik, Z. J., Skinner, J. E., Graf, K. E. \& Birbaumer, N. [1994] "Chaos and physiology: Deterministic chaos in excitable cell assemblies," Physiol. Rev. 74, 1-47.

Ellner, S. [1988] "Estimating attractor dimensions from limited data: A new method, with error estimates," Phys. Lett. A133, 128-133.

Farmer, J. D., Ott, E. \& York, J. A. [1983] "The dimension of chaotic attractors," Physica D7, 153-180.

Franaszek, M. \& Nabagło, A. [1993] "General case of crisis-induced intermittency in the Duffing equation," Phys. Lett. A178, 85-91.

Fraser, A. M., \& Swinney, H. L. [1986] "Independent coordinates for strange attractors from mutual information," Phys. Rev. A33, 1134-1140.

Fraser, A. M. [1989] "Reconstructing attractors from scalar time series: A comparison of singular system and redundancy criteria," Physica D34, 391-404.

Freeman, W. J. [1979] "EEG analysis gives model of neuronal template-matching mechanism for sensory search with olfactory bulb," Biol. Cybern. 35, 221-234.

Freeman, W. J. \& DiPrisco, V. [1986] "EEG spatial pattern differences with discriminated odors manifest chaotic and limit cycle attractors in olfactory bulb of rabbits," in Brain Theory, eds. Palm, G. \& Aertsen, A. (Springer-Verlag, Berlin, Heidelberg, New York), pp. 97-119.

Freeman, W. J. \&. Skarda, C. A. [1985] "A perspective on brain theory: Nonlinear dynamics of neural masses," Brain Res. Rev. 10, 147-175.

Fuchs, A. \& Kelso, J. A. S. [1992] "Self-organization in brain and behavior: Critical instabilities and dynamics of spatial model," in Nonlinear Dynamical Analysis of the EEG, eds. Jansen, B. H. \& Brandt, M. E. (World Scientific-Singapore), pp. 269-284.

Graf, K. \& Elbert, T. [1989] "Dimensional analysis of the waking EEG." in Brain Dynamics Progress and Perspectives, eds. Basar, E. \& Bullock, T. H. (SpringerVerlag, Berlin), pp. 174-191.

Grassberger, P. \& Procaccia, I. [1983a] "Measuring the strangeness of strange attractors," Physica D9, 189-208.

Grassberger, P. \& Procaccia, I. [1983b] "Estimating the Kolmogorov entropy from a chaotic signal," Phys. Rev. A28, 2591-2593. 
Grebogi, C., Ott, E. \& Yorke, J. A. [1982] "Chaotic attractors in crisis," Phys. Rev. Lett. 48, 1507-1510.

Grebogi, C., McDonald, S. W., Ott, E. \& Yorke, J. A. [1983] "Final state sensitivity: An obstruction to predictability," Phys. Lett. A99, 415-418.

Györgyi, G. \& Szèpfalusy, P. [1985] "Calculation of the entropy in chaotic systems," Phys. Rev. A31, 3477-3479.

Iasemidis, L. D. \& Sackellares, J. C. [1991] "The evolution with time of the spatial distribution of the largest Lyapunov exponent on the human epileptic cortex," in Measuring Chaos in the Human Brain, eds. Duke, D. \& Pritchard, W. (World Scientific, Singapore), pp. 97-112.

Kaplan, D. \& Glass, L. [1992] "Direct test for determinism in a time series," Phys. Rev. Lett 68, 427-430.

Kowalik, Z. J., Goerke, N., Bode, M. \& Purwins, H.-G., [1995a] "An experimental study of a driven Van-derPol-like Oscillator," submitted for publication.

Kowalik, Z. J., Franaszek, M. \& Pierański, P. [1988] "Selfreanimating chaos in the bouncing ball system," Phys. Rev. A37, 4016-4023.

Kowalik, Z. J., Elbert, T. \& Hoke, M. [1993] "Mapping dynamic brain function: The Largest Lyapunov exponent derived from multichannel magnetoencephalography," in Nonlinear Dynamical Analysis of the EEG, eds. Jansen, B. H. \& Brandt, M. E. (World Scientific, Singapore), pp. 156-164.

Kowalik, Z. J., Schiepek, G., Kumpf, K., Roberts, L. E. \& Elbert, T. [1995b] "Psychotherapy as a chaotic process. Part II: The application of nonlinear analysis methods on quasi time series of the client-therapist interaction: A nonstationary approach" Psychotherapy Research, in press.

Kumpf, K., Kowalik, Z. J., Braun, Ch. \& Miltner, W. [1995] "Measuring changes in brain dynamical activity," (submitted for publication).

Lai, Y.-C., Grebogi, C. \& Yorke, J. A. [1992] in Applied Chaos, eds. Kim, J. H. \& Stringer, J. (J. Wiley \& Sons Inc.), pp. 441-455.

Mayer-Kress, G. (ed.) [1986] Dimensions and Entropies in Chaotic Systems. Springer Series in Synergetics, Vol. 39, (Springer-Verlag, Berlin, Heidelberg, New York, Tokyo)

Mayer-Kress, G. \& Holzfuss, J. [1987a] "Analysis of the human electroencephalogram with methods from nonlinear dynamics," in Temporal Disorder in Human Oscillatory Systems, eds. Rensing, L., an der Heiden, U., Mackey, M. C. (Springer-Verlag Berlin, Heidelberg, New York, Tokyo), pp. 57-68.

Meyer-Kress, G. \& Layne, S. P. [1987b] "Dimensionality of the human electroencephalogram," in Perspectives in Biological Dynamics and Theoretical Medicine Ann. N. Y. Acad. Sci., eds. Koslov, K. H., Mandell, A. J. \& Schlesinger, M. F. 504, 62-87.

Nychka, D., Ellner, S., McCaffrey, D. \& Gallant, A. R. [1992] "Finding chaos in noisy systems," J. Roy. Stat.
Soc. B54, 399-426.

Paluš, M., Albrecht, V. \& Dvořák, I. [1993] "Informationtheoretic test for nonlinearity in time series," Phys. Lett. A175, 203-209.

Pesin, Ya. B. [1977] "Characteristic lyapunov exponents and smooth ergodic theory," Russian Math. Surveys 32, 55-114.

Rapp, P. E., Bashore, T., Martinerie, J., Albano, A., Zimmermann, I. \& Mees, A. [1990] "Dynamics of brain electrical activity," Brain Topography 2, 99-118.

Rosenstein, M. T., Collins, J. J. \& De Luca, C. J. [1993] "A practical method for calculating largest Lyapunov exponents from small data sets," Physica D65, $117-134$.

Sano, M. \& Sawada, Y. [1985] "Measurement of the Lyapunov spectrum from a chaotic time series," Phys. Rev. Lett. 55, 1082-1085.

Sato, S., Sano, M. \& Sawada, Y. [1987] "Practical methods of measuring the generalized dimension and the largest Lyapunov exponent in high dimensional chaotic systems," Progress in Theoretical Physics 77, 1-5.

Schiepek, G., Kowalik, Z. J., Schütz, A., Köhler, M., Richter, K., Strunk, G., Mühlnickel, W. \& Elbert, T. [1995] "Psychotherapy as a chaotic process. Part I: Coding the client-therapist interaction by means of sequential plan analysis and the search for chaos: A stationary approach," Psychotherapy Research, in press.

Schuster, H.-G. [1989] Deterministic Chaos. An Introduction (Verlag Chemie, Weinheim).

Skinner, J. E., Martin, J. L., Landisman, C. E., Mommer, M. M., Fulton, K., Mitra, M., Burton W. D. \& Saltzberg, B. [1990] "Chaotic attractors in a model of neocortex: dimensionalities of olfactory bulb surface potentials are spatially uniform and event-related," in Brain Dynamics, Progress and Perspectives, eds. Basar, E. \& Bullock, T. H. (Springer-Verlag, Berlin), pp. 119-134.

Skinner, J. E., M. Molnar, T. Vybiral \& Mitra, M. [1992] "Application of chaos theory to biology and medicine," Integrative Physiological and Behavioral Science 27, 39-53.

Soong, A. C. K. \& Stuart, C. I. J. M. [1989] "Evidence of chaotic dynamics underlying the human alpha-rhythm electroencephalogram," Biol. Cybern. 62, 55-62.

Takens, F. [1981] "Detecting strange attractors in turbulence" in Dynamical systems and turbulence, eds. Rand, D. A. \& Young, S. (Springer-Verlag, Berlin), pp. 366 .

Theiler, J., Eubank, S. Longtin, A., Galdrikian, B. \& Farmer, J.D. [1992] "Testing for nonlinearity in time series: the method of surrogate data," Physica D58, $77-94$.

Wolf, A., Swift, J. B., Swinney, H. L. \& Vastano, J. A. [1985] "Determining Lyapunov exponents from a time series," Physica D16, 285-317.

Wolff R. C. L. [1992] "Local Lyapunov exponents: Looking closely at chaos," J. Roy. Stat. Soc. B54, 353-371. 